\title{
Fatores associados à troca de sexo por dinheiro em homens que fazem sexo com homens no Brasil
}

\author{
Factors associated with exchanging sex for money in men who have \\ sex with men in Brazil
}

Denyr Jeferson Dutra Alecrim (https://orcid.org/0000-0003-0913-418X) ${ }^{1}$ Maria das Graças Braga Ceccato (https://orcid.org/0000-0002-4340-0659) ${ }^{1}$ Inês Dourado (https://orcid.org/0000-0003-1675-2146) ${ }^{2}$

Ligia Kerr (https://orcid.org/0000-0003-4941-408X) ${ }^{3}$

Ana Maria de Brito (https://orcid.org/0000-0001-6592-0762) ${ }^{4}$

Mark Drew Crosland Guimarães (https://orcid.org/0000-0001-7932-3854) ${ }^{5}$
${ }^{1}$ Faculdade de Farmácia, Universidade Federal de Minas Gerais (UFMG). Av. Antonio Carlos 6627, Pampulha. 31270-010 Belo Horizonte MG Brasil. denyrjeferson@gmail.com

${ }^{2}$ Instituto de Saúde Coletiva, Universidade Federal da Bahia. Salvador, BA, Brasil. ${ }^{3}$ Universidade

Federal do Ceará.

Departamento de Saúde

Comunitária. Fortaleza, CE, Brasil.

${ }^{4}$ Instituto Aggeu Magalhães, Fiocruz. Recife PE Brasil.

${ }^{5}$ Faculdade de Medicina,

UFMG. Belo Horizonte MG

Brasil.

\begin{abstract}
This study aimed to analyze the association between sociodemographic, programmatic and contextual factors and the receipt of money in exchange for sex among men who have sex with men (MSM). This is a multicenter, cross-sectional study conducted in ten Brazilian cities between 2008 and 2009. Adult MSM recruited through the Respondent Driven Sampling (RDS) were interviewed. Weighted Odds Ratio (ORw) was obtained through logistic regression, retaining the variables associated with the event $(p<0.05)$ in the final model. Of the total sample, 33.3\% reported receiving money in exchange for sex in the last 12 months before the interview. The variables that were independently associated with the event were age less than or equal to 25 years, lower education, lower social classes, previous history of syphilis, using sites or services to find sexual partners in the previous month, very high risk behavior, using illicit drugs in the previous six months, self-identifying as heterosexual or bisexual, having suffered physical violence due to sexual orientation and having suicidal thoughts always or most of the time. It was observed that MSM who received money in exchange for sex had greater socioeconomic, programmatic and contextual vulnerability, potentially increasing the risk of HIV infection than the other MSM in the sample.
\end{abstract}

Key words Men who have sex with men, Commercial sex, HIV
Resumo Objetivou-se analisar a associação entre fatores sociodemográficos, programáticos $e$ contextuais e ter recebido dinheiro em troca de sexo entre homens que fazem sexo com homens (HSH). Estudo multicêntrico, transversal, realizado em 10 cidades brasileiras, entre 2008 e 2009. Foram entrevistados 3.749 HSH selecionados pela técnica amostral "Respondent Driven-Sampling" (RDS). "Odds Ratio" ponderado $\left(\mathrm{OR}_{p}\right)$ foi obtido por meio de regressão logística, permanecendo no modelo final as variáveis associadas ao evento ( $p$ $<0,05)$. Da amostra total, 33,3\% relataram ter recebido dinheiro em troca de sexo nos últimos 12 meses anteriores à entrevista. As variáveis associadas com o evento foram ter idade $\leq 25$ anos, menor nivel de escolaridade, classes sociais mais baixas, história prévia de infecção por sífilis, utilizar locais de encontros para ter parceiros sexuais, ter comportamento de risco para HIV muito alto e usar drogas ilícitas, se autoidentificarem como heterossexual ou bissexual, ter sofrido violência física devido à orientação sexual e apresentar ideação suicida sempre ou na maioria das vezes. Observa-se que os HSH que receberam dinheiro em troca de sexo apresentaram maior vulnerabilidade socioeconômica, programática e contextual, potencialmente aumentando o risco de infecção pelo HIV em relação aos demais HSH.

Palavras-chave Homens que fazem sexo com homens, Sexo comercial, HIV 


\section{Introdução}

A infecção pelo HIV apresenta uma elevada prevalência entre os Homens que fazem Sexo com Homens (HSH) no Brasil representando 14,2\% em comparação a $0,6 \%$ na população geral adulta brasileira ${ }^{2}$. Quando comparada à taxa de infecção entre os homens heterossexuais, a relação é 13 vezes maior entre $\mathrm{HSH}$ residentes no Brasil ${ }^{1,3,4}$. Diversos estudos têm chamado atenção, de forma consistente, para a elevada prevalência de infecção pelo HIV entre HSH que trocam sexo por dinheiro, com estimativas que variam de $14 \%$ a $31 \%$ nos Estados Unidos da America ${ }^{5,6}$, de 4,1 a $24,4 \%$ na América do Sul ${ }^{7-11}$ e de 3,1 a $13,0 \%$ no Brasil $^{12,13}$.

O trabalho sexual é definido como a venda de serviços sexuais consensuais por adultos em troca de dinheiro, bens ou objetos ${ }^{14}$, podendo esta atividade ocorrer regularmente ou ocasionalmente e, conforme as legislações do país, formalmente ou informalmente ${ }^{15,16}$. Devido à associação do trabalho sexual a uma prática profissional com baixa aceitabilidade social e que carrega consigo características e signos sociais muitas vezes estigmatizados, os HSH descrevem a troca de sexo por dinheiro como uma atividade ocasional para se sustentar temporariamente ou pagar por um bem caro e não se identificando como trabalhadores do sexo ${ }^{11,16,17}$. Além disso, existe uma crescente tendência do trabalho sexual deixar de ser baseado nas experiências da rua e passar para o campo da internet, o que dificulta ainda mais a identificação destas pessoas como parte de um grupo social homogêneo ${ }^{18}$.

Dentre os fatores associados com a iniciação dos homens ao trabalho sexual, os mais comuns são os fatores econômicos, como a pobreza absoluta, $\mathrm{o}$ abandono familiar e aqueles associados a dificuldades para a integração ao mercado formal de trabalho, como a baixa escolaridade e qualificação profissional ${ }^{12,19-21}$. Os HSH que recebem dinheiro em troca de sexo podem possuir maior vulnerabilidade às doenças sexualmente transmissíveis (DST), devido a fatores não só relacionados ao elevado número de parceiros sexuais como também pelo envolvimento em práticas e situações associadas a baixo nível socioeconômico e às relações sexuais de risco, como uso de drogas e uso inconsistente de preservativos ${ }^{22}$. Uma recente revisão sistemática indica uma associação entre a troca de sexo por dinheiro e maior risco de infecção pelo HIV entre HSH de diversos países ${ }^{16}$.

O Plano Nacional de Enfrentamento da epidemia de AIDS e das DST entre gays, HSH e tra- $v e^{2}{ }^{23} s^{23}$ não faz nenhuma menção aos HSH que receberam dinheiro em troca de sexo, deixando evidente o silenciamento das ações em saúde direcionadas a esse segmento, que só aparecem de modo implícito na subcategoria "outros homens que fazem sexo com homens". Essa invisibilidade evidencia importante limitação do poder público em se adaptar, de forma apropriada, com ações de prevenção contextualizadas à complexidade das redes sexuais entre esses homens ${ }^{16}$. Os fatores de vulnerabilidade ao HIV/AIDS entre os HSH que receberam dinheiro em troca de sexo não devem ser considerados como problemas isolados, mas podem ser exemplos convincentes da necessidade de respostas abrangentes ao HIV que atendam às necessidades desse grupo diverso e complexo ${ }^{24,25}$. Seguindo uma nova estratégia de vigilância epidemiológica, informações sobre as populações sob maior vulnerabilidade ao HIV/ AIDS devem ser periodicamente investigadas, a fim de se construir um quadro informativo sobre a dinâmica da epidemia ao longo do tempo ${ }^{26}$.

Apesar de evidências indicarem que a troca de sexo por dinheiro entre HSH está potencialmente relacionada com fatores sociodemográficos, programáticos e contextuais, são limitados os estudos de abrangência nacional que abordam o tema. Além disto, grande parte dos estudos com esta população não distingue claramente aqueles que recebem ou pagam pela troca de sexo, estando os primeiros potencialmente em maior situação de risco ${ }^{16}$. As concepções, os valores e as práticas que organizam a construção da masculinidade, a adoção de representações e entendimentos sobre as práticas sexuais que adotam, que dificultam a percepção da vulnerabilidade às DST e HIV/AIDS, a vulnerabilidade de gênero, o baixo nível de conhecimento sobre DST e riscos do comportamento ou ligados ao indivíduo, a dificuldade de negociação sobre as práticas de prevenção com os clientes, dentre outros, são fatores que podem estar relacionados à menor admissão ou aceitação (apropriação) do risco vivenciado, o que leva a dificuldade na conscientização, mudança de comportamentos e minimização dos riscos ${ }^{20}$. Além dos fatores de vulnerabilidade ligados diretamente às DST e HIV/AIDS, outros fatores são bastante frequentes, como a exposição às potenciais violências, estigmatização do trabalho sexual e da homossexualidade, que pairam sobre os $\mathrm{HSH}$ que recebem dinheiro em troca de sexo $^{20}$.

É nesse contexto que se apresenta este estudo, que tem o objetivo de analisar a associação entre os fatores sociodemográficos, programáticos 


\section{Métodos}

Estudo de corte transversal, parte de uma pesquisa de abrangência nacional, realizado entre 2008 e 2009, com HSH residentes em dez cidades brasileiras que teve como objetivo estimar a prevalência da infecção por HIV e síflis e avaliar o conhecimento em HIV/AIDS, atitudes e práticas sexuais nesta população. Detalhes metodológicos do estudo estão disponíveis em Kerr et al. ${ }^{1}$. A técnica amostral utilizada para recrutar os $\mathrm{HSH}$ participantes foi o Respondent Driven Sampling (RDS), um método de amostragem por cadeia utilizado com populações de difícil acesso ${ }^{27}$. Foi realizado o cálculo amostral por cidade para fornecer estimativas independentes, resultando uma amostra de 250 a 350 HSH em cada cidade do estudo ${ }^{1}$. Desta forma, foram recrutados 3.746 HSH residentes nas cidades de Manaus, Recife, Salvador, Belo Horizonte, Rio de Janeiro, Santos, Curitiba, Itajaí, Brasília e Campo Grande.

Foi realizada uma pesquisa formativa utilizando métodos qualitativos para ajustar a logística e os protocolos de pesquisa ${ }^{28}$. Após selecionaremse os HSH da amostra inicial da população-alvo, denominados "sementes", os mesmos receberam três cupons numerados e com orientações sobre a pesquisa para convidar três HSH de sua rede social. Os cupons foram controlados por um sistema de gerenciamento. Os participantes selecionados como "sementes" que compareceram aos locais da pesquisa com o cupom válido (número de identificação e data de validade) e cumpriram os critérios de inclusão, constituíram a primeira onda da amostragem. Este processo se repetiu com seus convidados, até que a amostra atingisse o tamanho desejado em cada cidade.

HSH com idade igual ou superior a 18 anos e residentes nos municípios selecionados foram convidados a participar do estudo. Foram considerados elegíveis aqueles que cumpriram os seguintes critérios de inclusão: 1 . Ter tido pelo menos uma relação sexual com um homem nos últimos 12 meses; 2. Aceitar as condições para

participar do estudo, que incluem responder um questionário estruturado, assinando o Termo de Consentimento Livre e Esclarecido, e estar disposto a convidar seus pares a participarem do estudo; 3. Apresentar um cupom válido. Foram excluídos os participantes que se identificaram como travesti ou transexual. A pesquisa foi conduzida de acordo com a Resolução 466/12 Conselho Nacional de Saúde - Ministério da Saúde. O projeto foi aprovado pela Comissão Nacional de Ética em Pesquisa, pelo Comitê de Ética em Pesquisa da Universidade do Ceará e pelos serviços de saúde das cidades participantes ${ }^{1,28}$.

$\mathrm{O}$ evento de interesse foi ter recebido dinheiro em troca de sexo nos últimos 12 meses anteriores à entrevista considerando-se respostas positivas à pergunta feita durante a entrevista: "Nos últimos 12 meses, você recebeu dinheiro em troca de sexo?"

As potenciais variáveis de associação foram organizadas nos seguintes três blocos: 1 . sociodemográficas (idade, escolaridade, classe social, cor/raça, situação conjugal, e situação de trabalho no momento da entrevista); 2. programáticas (conhecimento sobre a transmissão do HIV, testagem prévia para HIV, positividade sorológica para HIV, testagem prévia para sífilis, história de sífilis, positividade sorológica para sífilis e, nos últimos 12 meses anteriores à entrevista, ter história de DST, receber aconselhamento sobre DST, receber preservativos gratuitos e receber gel lubrificante; 3. contextuais, subdivididas em: 3.a. comportamentais (gênero do primeiro parceiro sexual, idade da primeira relação sexual, número parceiros últimos seis meses, uso de preservativo em todas as relações sexuais nos últimos 12 meses, utilizar locais/serviços para encontrar parceiros no último mês, relações sexuais com participantes da rede de potenciais convidados, relação sexual sob efeito de qualquer droga últimos seis meses, autopercepção de risco de se infectar pelo HIV, escore de Comportamento de Risco referentes aos últimos 12 meses, frequência de uso de álcool, uso de drogas ilícitas nos últimos seis meses; 3.b relacionadas à orientação sexual - autoidentidade sexual, discriminação devido à orientação sexual, ter sofrido violência verbal, ter sofrido violência física, ter sofrido violência sexual; e $3 . c$ relacionadas à saúde mental - sentir-se tenso/preocupado, problemas para dormir, sentir medo e pânico, sentir-se triste/deprimido, ter ideação suicida. O conhecimento sobre transmissão do HIV foi mensurado por meio de 10 perguntas sobre transmissão e prevenção do HIV, sendo considerado conhecimento suficiente quando o 
participante acertou oito ou mais perguntas ${ }^{29}$. O escore de comportamento de risco para o HIV foi calculado a partir das informações do número de parceiros masculinos e uso de preservativos em relações anais nos últimos 12 meses com parceiros fixos, casuais e comerciais. O escore final foi calculado por meio da soma dos resultados obtidos, podendo variar de 0-48 pontos, sendo que valores altos indicam um maior grau de comportamento sexual de risco. Esse escore foi categorizado em baixo e médio risco ( $0-8$ pontos) e alto risco ( 9 pontos ou +$)^{29}$.

Os dados dos RDS foram ponderados de acordo com o tamanho da rede social e proporção de HSH em cada cidade relacionada à amostra total, baseado no método utilizado para o RDS por Szwarcwald et al. ${ }^{30}$. A magnitude da associação entre as variáveis explicativas e o evento foi estimada pelo Odds Ratio ponderado $\left(\mathrm{OR}_{\mathrm{p}}\right)$ com intervalo de 95\% de confiança (IC95\%). Para a análise de associação, utilizou-se regressão logística. A análise multivariada foi realizada, inicialmente, para cada bloco separadamente: 1 . características sociodemográficas; 2. programáticas; 3.a. comportamentais; 3.b. relacionadas à orientação sexual e; 3.c. relacionadas à saúde mental. Para cada bloco, a modelagem foi iniciada com variáveis estatisticamente associadas com o evento na análise univariada considerando um $\mathrm{p}$-valor $<0,20$. As variáveis explicativas foram deletadas sequencialmente dentro de cada bloco e, somente aquelas estatisticamente associadas com o evento, considerando um $\mathrm{p}$-valor $<0,10$, permaneceram nos modelos intermediários. A modelagem final foi iniciada com aquelas variáveis estatisticamente significantes $(\mathrm{p}<0,10) \mathrm{em}$ cada modelo intermediário seguida de deleção sequencial. Somente aquelas associadas com o evento com $p$-valor $<0,05$ permaneceram no modelo final. As análises foram realizadas utilizando o software SAS ${ }^{\circledast}$ (SAS Inst., Cary, USA).

\section{Resultados}

Foram recrutados 3859 HSH participantes nas dez cidades brasileiras. Destes, 3749 tinham informações disponíveis sobre sexo comercial e foram incluídos nesta análise. Deste grupo, 1146 $(33,3 \%)$ relataram ter recebido dinheiro em troca de sexo nos 12 meses anteriores às entrevistas. Mais da metade da amostra foi constituída por HSH acima de 25 anos (58,4\%), com mais de oito anos de estudo (58,6\%), pertencentes às classes econômicas mais baixas (C-D-E), e a maior parte era de cor/raça não branca $(83,2 \%)$, solteira ou vivendo sozinha $(84,4 \%)$. Aproximadamente $30 \%$ não estavam trabalhando no momento da entrevista (Tabela 1).

Em relação às variáveis programáticas, o conhecimento sobre prevenção e transmissão do HIV foi insuficiente, para $42,3 \%$ dos entrevistados. Cerca de metade dos indivíduos $(48,4 \%)$ já haviam feito teste sorológico para HIV alguma vez na vida e no teste sorológico para HIV realizado na pesquisa, $12,6 \%$ foram positivos. Por outro lado, apenas um quarto $(25,7 \%)$ já havia feito teste diagnóstico para sífilis, e 7,7\% possuem história prévia de sífilis, ambos alguma vez na vida. No teste sorológico para sífilis realizado na pesquisa, $14,6 \%$ foram positivos. Uma grande proporção $(72,4 \%)$ recebeu preservativos de forma gratuita nos 12 meses anteriores e somente $45,5 \%$ destes receberam gel lubrificante no mesmo período (Tabela 1 ).

Dentre as variáveis contextuais, no que diz respeito ao comportamento sexual, a primeira relação sexual foi com parceira mulher em 52,9\% dos entrevistados e quase a metade $(46,9 \%)$ iniciou a atividade sexual com idade igual ou inferior a 14 anos. Nos 12 meses anteriores à entrevista, cerca de um quarto dos participantes $(24,5 \%)$ relatou ter tido mais de cinco parceiros sexuais. Quanto ao uso de drogas, $42,8 \%$ informaram uso de alguma droga ilícita durante os seis meses anteriores à entrevista. Cerca de $20 \%$ dos HSH entrevistados foram classificados com alto comportamento de risco para o HIV.

Quanto à orientação sexual, aproximadamente $39 \%$ se autoidentificaram como bissexual ou heterossexual e $43,3 \%$ e $13,4 \%$ sofreram violência verbal e física devido à orientação sexual, respectivamente com relato de violência sexual por $15,0 \%$.

Em relação à saúde mental, informaram que, durante os seis meses anteriores à entrevista sempre ou na maioria das vezes sentiram-se tenso ou preocupado $(74,1 \%)$, triste ou deprimido $(54,2 \%)$, com problemas para dormir $(42,5 \%)$, medo ou pânico $(22,2 \%)$. Além disso, $11,0 \%$ dos participantes informaram ter pensamento suicida sempre, ou na maioria das vezes.

A análise univariada indicou que a maioria das variáveis apresentou associação estatisticamente significante com o evento (Tabela 2).

No modelo final de regressão logística, estiveram associadas de forma independente com ter recebido dinheiro por sexo 12 meses anteriores a entrevista (Tabela 3 ): ser jovem (idade menor ou igual a 25 anos) $(\mathrm{ORp}=2,04)$; possuir me- 
Tabela 1. Características descritivas da amostra de homens que fazem sexo com homens, Brasil, 2009. $(\mathrm{N}=$ $\left.3749^{1}\right)$.

\begin{tabular}{|c|c|c|}
\hline Características & $\mathbf{n}^{2}(\%)$ & $\%_{p}^{3}$ \\
\hline \multicolumn{3}{|l|}{ a. Sociodemográficas } \\
\hline Idade ( $\leq 25$ anos): & $1971(52,8)$ & 41,6 \\
\hline Escolaridade ( $\leq 8$ anos): & $1127(30,2)$ & 41,4 \\
\hline Classe Social (C-D-E): & $2563(71,2)$ & 75,2 \\
\hline Cor/Raça (Não branco): & $3275(87,4)$ & 83,2 \\
\hline Situação conjugal (Solteiro/Sozinho): & $3128(83,5)$ & 84,4 \\
\hline Situação de trabalho atual (Não): & $1208(32,2)$ & 29,5 \\
\hline \multicolumn{3}{|l|}{ b. Programáticas } \\
\hline Positividade sorológica para HIV (Sim): & $323(9,8)$ & 12,6 \\
\hline Positividade sorológica para sífilis (Sim): & $340(10,3)$ & 14,6 \\
\hline Conhecimento sobre a transmissão do HIV (Insuficiente): & $1617(43,2)$ & 42,3 \\
\hline Testagem prévia para HIV (Nunca): & $1777(47,4)$ & 48,4 \\
\hline Testagem prévia para sífilis (Nunca): & $2579(71,4)$ & 74,3 \\
\hline História prévia de sífilis (Sim): & $232(6,2)$ & 7,7 \\
\hline História de DST nos últimos 12 meses (Sim): & $854(22,8)$ & 27,6 \\
\hline Recebeu preservativos gratuitos últimos 12 meses (Não): & $873(23,3)$ & 27,6 \\
\hline Recebeu aconselhamento DST últimos 12 meses (Não): & $1363(36,4)$ & 68,1 \\
\hline Recebeu gel lubrificante últimos 12 meses (Não): & $3099(82,7)$ & 54,5 \\
\hline \multicolumn{3}{|l|}{ iii. Contextuais: } \\
\hline \multicolumn{3}{|l|}{ iii.a. Comportamentais } \\
\hline Gênero do primeiro parceiro sexual (Mulher): & $1782(47,6)$ & 52,9 \\
\hline Idade primeira relação ( $\leq 14$ anos): & $1858(49,9)$ & 46,9 \\
\hline Número parceiros últimos 6 meses $(>5)$ : & $1078(29,0)$ & 24,5 \\
\hline Uso preservativo todas relações últimos 12 meses (Não): & $2241(59,8)$ & 63,6 \\
\hline Utilizar locais/serviços para encontrar parceiros último mês (Sim): & $1568(41,9)$ & 41,9 \\
\hline Sexo com rede de potenciais convidados (Sim): & $2207(61,0)$ & 51,8 \\
\hline Ter recebido dinheiro por sexo (Sim): & $1146(30,6)$ & 33,3 \\
\hline Relação sexual sob efeito de qualquer droga últimos 6 meses (Sim) & $2447(65,5)$ & 66,3 \\
\hline Auto percepção de risco de se infectar pelo HIV (Mod-Alta): & $1010(26,9)$ & 29,9 \\
\hline Escore de Comportamento de Risco - 12 meses ( $>8$ Muito Alto): & $738(19,7)$ & 19,9 \\
\hline Frequência de uso de álcool (2+Vezes por semana) & $2238(59,8)$ & 63,5 \\
\hline Uso de drogas ilícitas nos 6 meses (Sim): & $1497(40,1)$ & 42,8 \\
\hline \multicolumn{3}{|l|}{ iii.b. Relacionadas à orientação sexual: } \\
\hline Auto identidade sexual (Bi-Hetero): & $1450(38,7)$ & 38,7 \\
\hline Discriminação devido à Orientação Sexual (Sim) & $1293(36,4)$ & 27,9 \\
\hline Ter sofrido violência verbal (Sim): & $1697(45,3)$ & 43,2 \\
\hline Ter sofrido violência física (Sim): & $557(14,9)$ & 13,4 \\
\hline Ter sofrido violência sexual (Sim): & $592(15,8)$ & 15,0 \\
\hline \multicolumn{3}{|l|}{ iii.c. Relacionadas à saúde mental: } \\
\hline Sentir-se tenso/preocupado (Sempre/Maioria das vezes): & $2475(66,1)$ & 74,1 \\
\hline Problemas para dormir (Sempre/Maioria das vezes): & $1445(38,6)$ & 42,5 \\
\hline Sentir medo e pânico (Sempre/Maioria das vezes): & $731(19,5)$ & 22,2 \\
\hline Sentir-se triste/deprimido (Sempre/Maioria das vezes): & $1821(48,6)$ & 54,2 \\
\hline Ideação suicida (Sempre/Maioria das vezes): & $357(9,5)$ & 11,0 \\
\hline
\end{tabular}

nor nível de escolaridade $(\mathrm{ORp}=3,58)$; ser das classes sociais mais baixas (C-D-E) $(\mathrm{ORp}=2,35)$; ter história prévia de sífilis $(\mathrm{ORp}=1,60)$; ter tido primeira relação sexual com mulher (ORp $=2,32$ ) e com idade menor ou igual à 14 anos $(\mathrm{ORp}=1,78)$; ter tido relação sexual com mais 
Tabela 2. Análise Univariada dos fatores associados com receber dinheiro por sexo nos 12 meses anteriores, Brasil, 2009. $\left(\mathrm{N}=3749^{1}\right)$.

\begin{tabular}{|c|c|c|c|c|}
\hline Características & $\begin{array}{c}\text { SIM } \\
\mathrm{n}(\%)\end{array}$ & $\begin{array}{l}\mathrm{NÃO} \\
\mathrm{n}(\%)\end{array}$ & $\mathrm{OR}_{\mathrm{p}}^{2}(\mathrm{IC} 95 \%)$ & $\begin{array}{c}X^{2} \\
\text { (Valor-p) }\end{array}$ \\
\hline \multicolumn{5}{|l|}{ i. Sociodemográficas: } \\
\hline \multicolumn{5}{|l|}{ Idade: } \\
\hline$\leq 25$ anos & $676(34,9)$ & $1295(65,1)$ & $1,12(0,98-1,28)$ & 0,088 \\
\hline$>25$ anos & $464(32,3)$ & $1299(67,7)$ & 1,00 & \\
\hline \multicolumn{5}{|l|}{ Escolaridade: } \\
\hline$\leq 8$ anos & $563(52,6)$ & $564(47,4)$ & $4,50(3,90-5,20)$ & $<0,001$ \\
\hline$>9$ anos & $577(19,8)$ & $2029(80,2)$ & 1,00 & \\
\hline \multicolumn{5}{|l|}{ Classe Social: } \\
\hline C-D-E & $889(35,1)$ & $1674(64,9)$ & $4,27(3,41-5,37)$ & $<0,001$ \\
\hline A-B & $156(11,2)$ & $882(88,8)$ & 1,00 & \\
\hline \multicolumn{5}{|l|}{ Cor/Raça: } \\
\hline Não branco & $1012(33,1)$ & $2263(66,9)$ & $0,92(0,78-1,11)$ & 0,414 \\
\hline Branco & $134(34,7)$ & $338(65,3)$ & 1,00 & \\
\hline \multicolumn{5}{|l|}{ Situação conjugal: } \\
\hline Solteiro/Sozinho & $986(34,0)$ & $2146(66,0)$ & $1,18(0,98-1,43)$ & 0,076 \\
\hline Casado/União & $164(30,2)$ & $456(69,8)$ & 1,00 & \\
\hline \multicolumn{5}{|l|}{ Situação de trabalho atual: } \\
\hline Não & $446(36,8)$ & $762(63,2)$ & $1,24(1,07-1,44)$ & 0,003 \\
\hline Sim & $700(31,9)$ & $1841(68,1)$ & 1,00 & \\
\hline \multicolumn{5}{|l|}{ ii. Programáticas: } \\
\hline \multicolumn{5}{|l|}{ Positividade sorológica para HIV: } \\
\hline Não & $244(12,8)$ & $2029(87,2)$ & $0,94(0,76-1,16)$ & 0,547 \\
\hline Sim & $79(12,1)$ & $930(87,9)$ & 1,00 & \\
\hline \multicolumn{5}{|l|}{ Positividade sorológica para sífilis: } \\
\hline Não & $236(13,6)$ & $2055(86,4)$ & $1,29(1,06-1,56)$ & 0,011 \\
\hline Sim & $104(16,8)$ & $903(83,2)$ & 1,00 & \\
\hline \multicolumn{5}{|c|}{ Conhecimento sobre a transmissão do HIV: } \\
\hline Insuficiente & $601(38,8)$ & $1016(61,2)$ & $1,51(1,33-1,74)$ & $<0,001$ \\
\hline Suficiente & $542(29,5)$ & $1583(70,5)$ & 1,00 & \\
\hline \multicolumn{5}{|l|}{ Testagem prévia para HIV: } \\
\hline Não & $712(40,1)$ & $1065(59,9)$ & $1,81(1,58-2,07)$ & $<0,001$ \\
\hline $\operatorname{Sim}$ & $434(27,0)$ & $1538(73,0)$ & 1,00 & \\
\hline \multicolumn{5}{|l|}{ Testagem prévia para sífilis: } \\
\hline Não & $901(35,1)$ & $1678(64,9)$ & $1,37(1,17-1,61)$ & $<0,001$ \\
\hline $\operatorname{Sim}$ & $209(28,3)$ & $824(71,7)$ & 1,00 & \\
\hline \multicolumn{5}{|l|}{ História prévia de sífilis: } \\
\hline Sim & $62(39,0)$ & $170(61,0)$ & $1,30(1,02-1,66)$ & $<0,034$ \\
\hline Não & $1081(33,0)$ & $2430(67,0)$ & & \\
\hline \multicolumn{5}{|c|}{ História de DST nos últimos 12 meses: } \\
\hline Sim & $281(40,3)$ & $573(59,7)$ & $1,52(1,31-1,76)$ & $<0,001$ \\
\hline Não & $862(30,8)$ & $2027(69,2)$ & 1,00 & \\
\hline \multicolumn{5}{|c|}{ Recebeu preservativos gratuitos últimos 12 meses: } \\
\hline Não & $267(32,0)$ & $606(68,0)$ & $0,92(0,78-1,07)$ & 0,257 \\
\hline $\operatorname{Sim}$ & $876(34,0)$ & $1993(66,0)$ & 1,00 & \\
\hline \multicolumn{5}{|c|}{ Recebeu aconselhamento DST últimos 12 meses: } \\
\hline Não & $363(27,9)$ & $1000(72,1)$ & $0,68(0,59-0,79)$ & $<0,001$ \\
\hline Sim & $781(36,0)$ & $1600(64,0)$ & 1,00 & \\
\hline
\end{tabular}


Tabela 2. Análise Univariada dos fatores associados com receber dinheiro por sexo nos 12 meses anteriores, Brasil, 2009. $\left(\mathrm{N}=3749^{1}\right)$.

\begin{tabular}{|c|c|c|c|c|}
\hline Características & $\begin{array}{c}\text { SIM } \\
\mathrm{n}(\%)\end{array}$ & $\begin{array}{l}\mathrm{NA} \tilde{\mathrm{O}} \\
\mathrm{n}(\%)\end{array}$ & $\mathrm{OR}_{\mathrm{p}}^{2}(\mathrm{IC} 95 \%)$ & $\begin{array}{c}X^{2} \\
(\text { Valor-p) }\end{array}$ \\
\hline \multicolumn{5}{|c|}{ Recebeu gel lubrificante últimos 12 meses: } \\
\hline Não & $1014(36,1)$ & $2085(63,9)$ & $2,40(1,94-2,99)$ & $<0,001$ \\
\hline Sim & $129(19,0)$ & $515(81,0)$ & 1,00 & \\
\hline \multicolumn{5}{|l|}{ iii. Contextuais } \\
\hline \multicolumn{5}{|l|}{ iii.a. Comportamentais: } \\
\hline \multicolumn{5}{|l|}{ Gênero do primeiro parceiro sexual: } \\
\hline Mulher & $731(42,7)$ & $1051(57,3)$ & $2,52(2,18-2,90)$ & $<0,001$ \\
\hline Homem/Travesti & $415(22,8)$ & $1548(77,2)$ & 1,00 & \\
\hline \multicolumn{5}{|l|}{ Idade primeira relação: } \\
\hline$\leq 14$ anos & $669(41,7)$ & $1189(58,3)$ & $2,01(1,75-2,30)$ & $<0,001$ \\
\hline$>14$ anos & $473(26,2)$ & $1394(73,8)$ & 1,00 & \\
\hline \multicolumn{5}{|c|}{ Número parceiros 6 meses anteriores: } \\
\hline$>5$ & $569(56,5)$ & $509(43,5)$ & $3,76(2,23-4,39)$ & $<0,001$ \\
\hline$\leq 5$ & $557(25,6)$ & $2082(74,4)$ & 1,00 & \\
\hline \multicolumn{5}{|c|}{$\begin{array}{l}\text { Uso preservativo todas relações } 12 \text { meses } \\
\text { anteriores: }\end{array}$} \\
\hline Não & $656(32,8)$ & $1595(67,2)$ & $0,93(0,82-1,08)$ & 0,371 \\
\hline Sim & $500(34,2)$ & $1008(65,8)$ & 1,00 & \\
\hline \multicolumn{5}{|c|}{$\begin{array}{l}\text { Utilizar locais ou serviços para encontrar } \\
\text { parceiros no mês anterior: }\end{array}$} \\
\hline Sim & $593(41,5)$ & $975(58,5)$ & $1,86(1,62-2,13)$ & $<0,001$ \\
\hline Não & $551(27,6)$ & $1625(72,4)$ & 1,00 & \\
\hline \multicolumn{5}{|c|}{ Sexo com rede de potenciais convidados: } \\
\hline Sim & $706(36,4)$ & $1501(63,6)$ & $1,32(1,16-1,52)$ & $<0,001$ \\
\hline Não & $401(30,1)$ & $1007(69,9)$ & 1,00 & \\
\hline \multicolumn{5}{|c|}{$\begin{array}{l}\text { Relação sexual sob efeito de qualquer droga nos } 6 \\
\text { meses anteriores: }\end{array}$} \\
\hline Sim & $879(38,2)$ & $1568(61,8)$ & $1,97(1,69-2,29)$ & $<0,001$ \\
\hline Não & $263(24,0)$ & $1025(76,0)$ & 1,00 & \\
\hline \multicolumn{5}{|c|}{ Auto percepção de risco de se infectar pelo HIV: } \\
\hline Moderada - Alta & $390(41,3)$ & $620(58,7)$ & $1,79(1,48-2,03)$ & 0,027 \\
\hline Não Sabe - ND & $211(32,2)$ & $454(67,8)$ & $1,17(0,99-1,40)$ & \\
\hline Nenhuma - Pouca & $545(28,8)$ & $1529(71,2)$ & 1,00 & \\
\hline \multicolumn{5}{|c|}{$\begin{array}{l}\text { Escore de Comportamento de Risco - } 12 \text { meses } \\
\text { anteriores: }\end{array}$} \\
\hline$>8$ (Muito Alto) & $358(54,9)$ & $353(45,1)$ & $3,16(2,69-3,72)$ & $<0,001$ \\
\hline$\leq 8$ (Baixo - Médio - Alto $)$ & $751(27,8)$ & $2249(72,2)$ & 1,00 & \\
\hline \multicolumn{5}{|l|}{ Frequência de uso de álcool: } \\
\hline 2 ou mais vezes por semana & $775(36,9)$ & $1463(63,1)$ & $1,55(1,34-1,79)$ & $<0,001$ \\
\hline Nunca/Eventual & $369(27,4)$ & $1135(72,6)$ & 1,00 & \\
\hline \multicolumn{5}{|c|}{ Uso de drogas ilícitas nos 6 meses anteriores: } \\
\hline Sim & $689(50,4)$ & $808(49,6)$ & $3,88(3,37-4,47)$ & $<0,001$ \\
\hline Não & $454(20,8)$ & $1784(79,2)$ & 1,00 & \\
\hline \multicolumn{5}{|c|}{ iii.b. Relacionadas à orientação sexual: } \\
\hline \multicolumn{5}{|c|}{ Auto identidade sexual: } \\
\hline Bissexual/Heterossexual & $690(48,6)$ & $760(51,4)$ & $3,05(2,66-3,51)$ & $<0,001$ \\
\hline HSH/Homossexual & $454(23,7)$ & $1841(76,3)$ & 1,00 & \\
\hline
\end{tabular}


Tabela 2. Análise Univariada dos fatores associados com receber dinheiro por sexo nos 12 meses anteriores, Brasil, 2009. $\left(\mathrm{N}=3749^{1}\right)$.

\begin{tabular}{|c|c|c|c|c|}
\hline Características & $\begin{array}{c}\text { SIM } \\
\mathrm{n}(\%)\end{array}$ & $\begin{array}{l}\mathrm{NA} O \\
\mathrm{n}(\%)\end{array}$ & $\mathrm{OR}_{\mathrm{p}}^{2}(\mathrm{IC} 95 \%)$ & $\begin{array}{c}X^{2} \\
\text { (Valor-p) }\end{array}$ \\
\hline \multicolumn{5}{|c|}{ Discriminação devido à Orientação Sexual: } \\
\hline Sim & $327(25,6)$ & $966(74,4)$ & $0,63(0,52-0,71)$ & $<0,001$ \\
\hline Não & $819(36,3)$ & $1637(63,7)$ & 1,00 & \\
\hline \multicolumn{5}{|l|}{ Ter sofrido violência verbal: } \\
\hline Sim & $459(28,6)$ & $1238(71,4)$ & $0,68(0,59-0,78)$ & $<0,001$ \\
\hline Não & $684(37,1)$ & $1362(62,9)$ & & \\
\hline \multicolumn{5}{|l|}{ Ter sofrido violência física: } \\
\hline Sim & $193(41,5)$ & $364(58,5)$ & $1,49(1,23-1,80)$ & $<0,001$ \\
\hline Não & $950(32,2)$ & $2235(67,8)$ & & \\
\hline \multicolumn{5}{|l|}{ Ter sofrido violência sexual: } \\
\hline $\operatorname{Sim}$ & $179(39,2)$ & $413(60,8)$ & $1,34(1,22-1,62)$ & 0,001 \\
\hline Não & $967(32,3)$ & $2190(67,7)$ & 1,00 & \\
\hline \multicolumn{5}{|l|}{ iii.c. Relacionadas à saúde mental: } \\
\hline \multicolumn{5}{|l|}{ Sentir-se tenso ou preocupado: } \\
\hline Sempre/Maioria das vezes & $759(33,4)$ & $1716(66,6)$ & $0,99(0,85-1,16)$ & 0,923 \\
\hline Nunca/Raramente & $385(33,6)$ & $883(66,4)$ & 1,00 & \\
\hline \multicolumn{5}{|l|}{ Problemas para dormir: } \\
\hline Sempre/Maioria das vezes & $488(35,7)$ & $957(64,3)$ & $1,19(1,04-1,37)$ & 0,010 \\
\hline Nunca/Raramente & $656(31,8)$ & $1642(68,2)$ & 1,00 & \\
\hline \multicolumn{5}{|l|}{ Sentir medo e pânico: } \\
\hline Sempre/Maioria das vezes & $308(44,0)$ & $423(56,0)$ & $1,80(1,54-2,11)$ & $<0,001$ \\
\hline Nunca/Raramente & $836(30,4)$ & $2176(69,6)$ & 1,00 & \\
\hline \multicolumn{5}{|l|}{ Sentir-se triste ou deprimido: } \\
\hline Sempre/Maioria das vezes & $617(37,5)$ & $1204(62,5)$ & $1,50(1,31-1,72)$ & $<0,001$ \\
\hline Nunca/Raramente & $527(28,6)$ & $1395(71,4)$ & 1,00 & \\
\hline \multicolumn{5}{|l|}{ Ideação suicida: } \\
\hline Sempre/Maioria das vezes & $173(59,8)$ & $184(40,2)$ & $3,44(2,79-4,24)$ & $<0,001$ \\
\hline Nunca/Raramente & $971(30,2)$ & $2414(69,8)$ & 1,00 & \\
\hline
\end{tabular}

${ }^{1}$ Os valores variam devido às informações ignoradas.

${ }^{2}$ Odds Ratio ponderado.

de cinco parceiros nos seis meses anteriores à entrevista $(\mathrm{ORp}=3,27)$; utilizar locais ou serviços para encontrar parceiros sexuais no mês anterior à entrevista $(\mathrm{ORp}=1,27)$; ter relações sexuais com os possíveis convidados para participar do estudo (ORp = 1,25); possuir comportamento de risco muito alto $(\mathrm{ORp}=2,76)$; usar drogas ilícitas nos seis meses anteriores à entrevista $(\mathrm{ORp}=$ 1,89 ); se autoidentificar como heterossexual ou bissexual $(\mathrm{ORp}=2,49)$; ter sofrido violência físi$\mathrm{ca}(\mathrm{ORp}=1,38)$ e não ter sofrido violência verbal $(\mathrm{ORp}=0,66)$ devido à orientação sexual e apresentar ideação suicida sempre ou na maioria das vezes $(\mathrm{ORp}=2,71)$.

\section{Discussão}

Os resultados obtidos indicam elevada proporção $(33,3 \%)$ de recebimento de dinheiro em troca de sexo no ano anterior à entrevista entre a população de HSH no Brasil. Os resultados encontrados neste estudo estão dentro da variação da proporção relatada na literatura nacional e internacional. Observa-se que, internacionalmente, há grande variação entre os resultados encontrados, de $16 \%$ a $63 \%$, que pode ser atribuída às diferentes características da população alvo, à sobreposição de fatores de vulnerabilidades, às diferenças de recrutamento, dentre outras ${ }^{31-35}$. No Brasil, na cidade de Campinas, foi encontrado que $14,8 \%$ dos HSH receberam dinheiro por sexo nos dois meses anteriores ${ }^{13}$. Desta forma, 


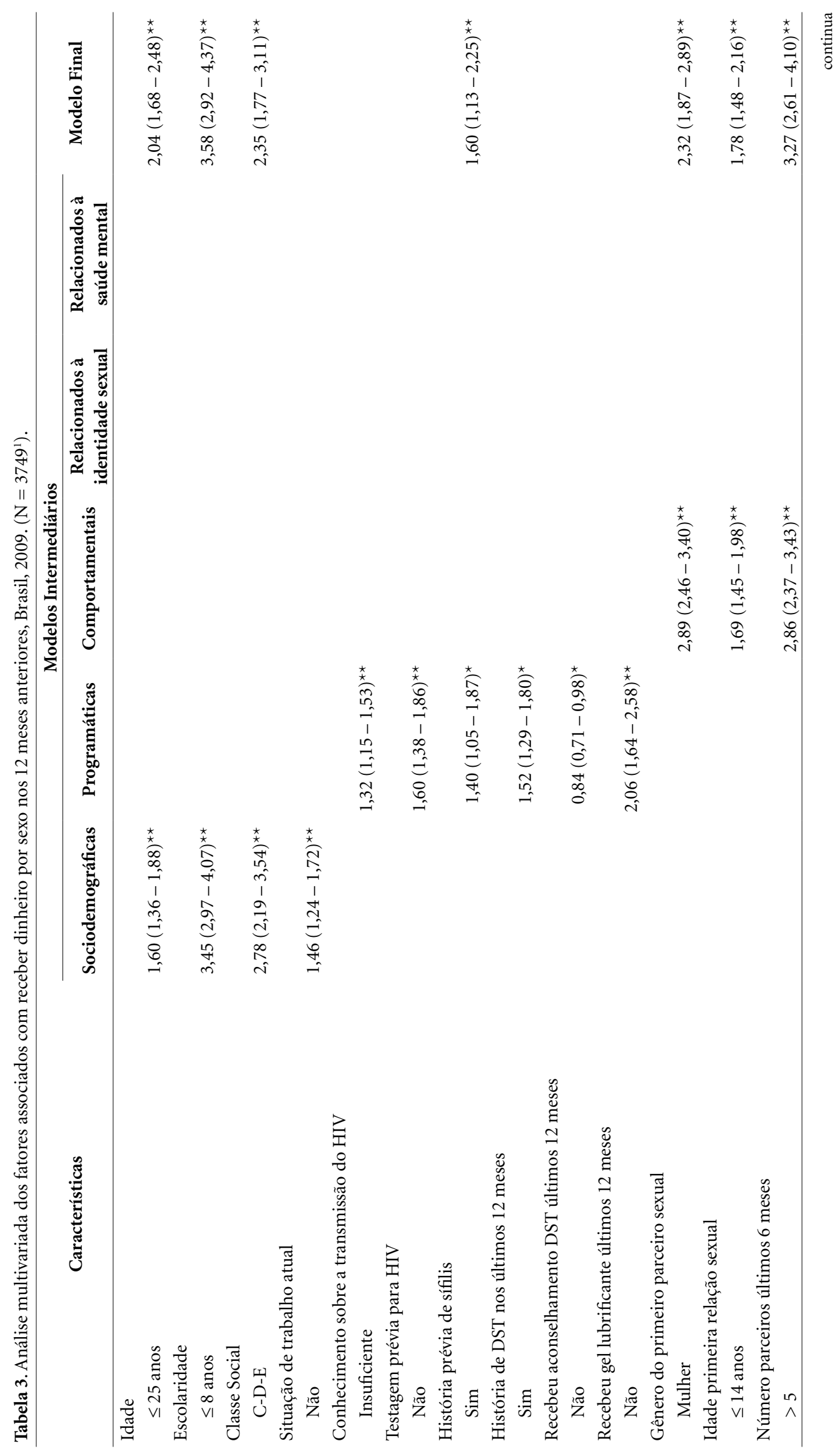




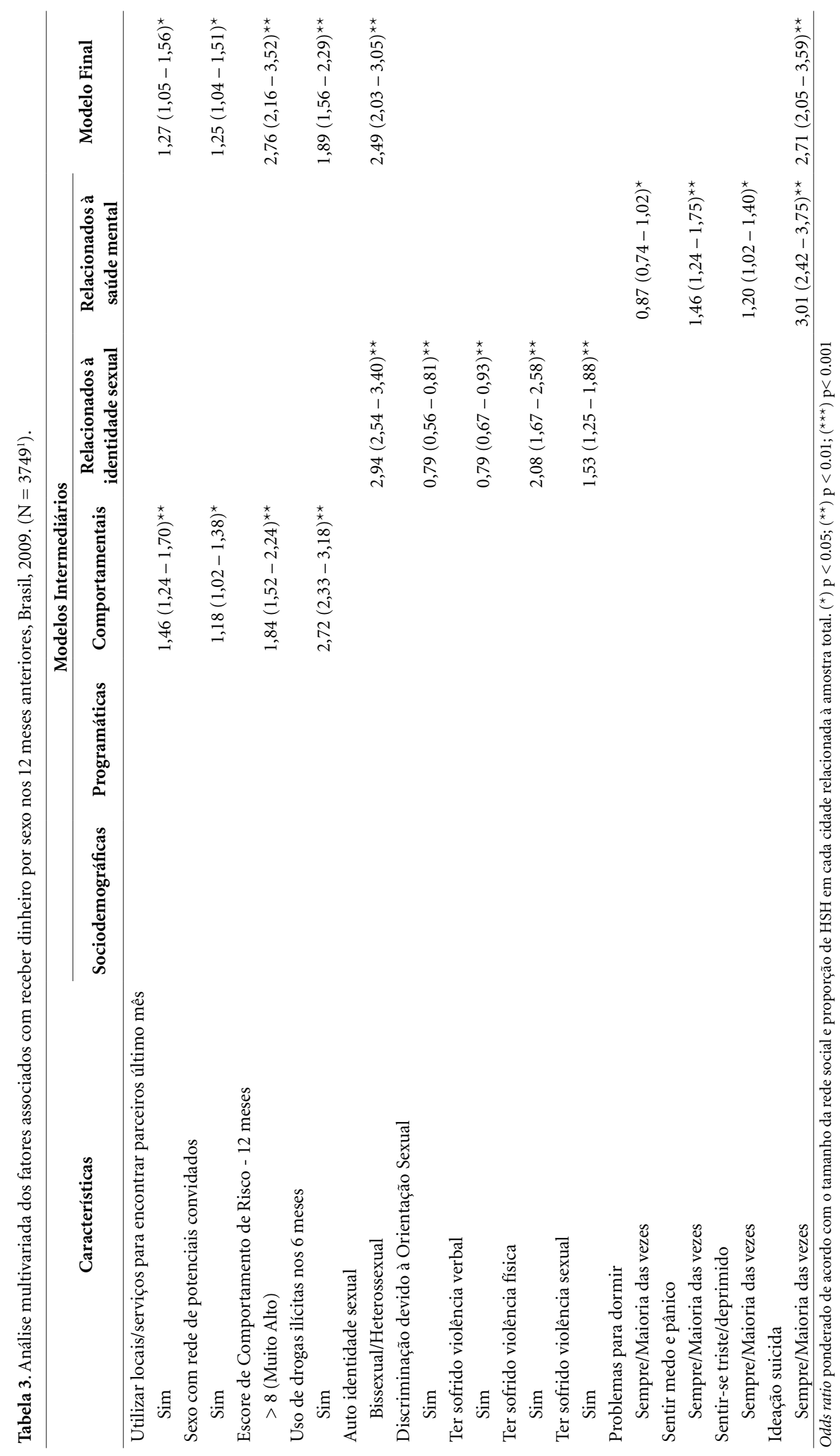


a comparação entre os resultados encontrados deve ser cautelosa, pois em sua maioria são limitados a estudos locais e com subpopulações de HSH em maior vulnerabilidade social como usuários de drogas, pessoas em situação de rua e desempregados. Assim, as diferenças metodológicas podem influenciar o resultado encontrado, pois metodologias em que o recrutamento é realizado pelo participante baseado em incentivo financeiro pode resultar em maior participação daqueles interessados nesse incentivo. Outro fator importante é a definição do tempo limite para a prática sexual em troca de dinheiro antes da entrevista, em 12 meses anteriores nesse estudo e de até dois meses em Tun et al. ${ }^{13}$.

Os $\mathrm{HSH}$ que receberam dinheiro em troca de sexo neste estudo apresentaram importantes diferenças no que tange às questões sociodemográficas quando comparados aos demais $\mathrm{HSH}$. Os $\mathrm{HSH}$ que receberam dinheiro em troca de sexo apresentaram menor escolaridade, classes sociais mais baixas e menores chances de estarem empregados no momento da entrevista. Esses resultados são consistentes com outros estudos realizados no Brasil e em outros países ${ }^{6,12,13,32}$. Seguindo a tendência internacional, nesta amostra os HSH que receberam dinheiro em troca de sexo apresentam maior marginalização social quando comparados aos demais HSH. A marginalização desse grupo pode levar a violações de direitos humanos resultando em barreiras substanciais ao acesso dessas pessoas às informações de prevenção e aos serviços de saúde para a prevenção e o tratamento da infecção pelo $\mathrm{HIV}^{36}$.

A associação positiva entre receber dinheiro em troca de sexo e ser mais jovem (idade menor ou igual a 25 anos) também é descrita por outros estudos ${ }^{6,12,32,37}$. Apesar das complexidades éticas envolvidas em pesquisas envolvendo jovens e adolescentes, resultando em poucos estudos com esta população, muitos HSH que receberam dinheiro em troca de sexo, em diferentes países, relatam terem iniciado o trabalho sexual na adolescência, em algumas vezes em condições de coerção ou força ${ }^{38}$ devido ao fator econômico ou abandono familiar ${ }^{19}$. A elevada prevalência de infecção pelo HIV observada entre HSH na adolescência e juventude pode sugerir que os fatores de vulnerabilidade presentes durante a adolescência estejam relacionados com a ocorrência da infecção ${ }^{16}$. Parcela importante dos HSH que receberam dinheiro em troca de sexo deste estudo relataram primeira relação sexual com até 14 anos e com parceiras mulheres. Apesar do aumento no uso de preservativo na primeira relação sexual entre jovens de 16 a 19 anos, no Brasil, há um crescimento da atividade sexual sem preservativo entre os jovens que começaram a vida sexual antes dos 14 anos $^{39}$. Além disso, a iniciação sexual mais tardia, ou seja, mais de 17 anos para os rapazes, é importante para a determinação do uso subsequente de preservativo ${ }^{40}$. Quanto mais precoce o início da atividade sexual entre membros da população de $\mathrm{HSH}$, maior a chance deste indivíduo manter comportamento sexual de risco para infecção pelo HIV na vida adulta ${ }^{29}$.

Nos últimos anos, o uso da internet e de aplicativos de rede geosocial para telefones com o objetivo de encontrar parceiros sexuais ganhou destaque entre os $\mathrm{HSH}$ em todo o mundo ${ }^{41,42}$. Além disso, existem espaços específicos de socialização entre os membros da população de $\mathrm{HSH}$, como bares e boates caracterizados como LGBT ou "gay-friendly", praças, parques, saunas, dentre outros. Neste estudo, ter recebido dinheiro em troca de sexo esteve associado de forma positiva com utilizar locais ou serviços para encontrar parceiros sexuais no mês anterior. Observa-se então, uma tendência do trabalho sexual também estar relacionado com experiências no campo da internet e aplicativos de rede geosocial, além daqueles já usualmente identificados ${ }^{18}$.

Neste estudo encontrou-se que os HSH que receberam dinheiro em troca de sexo relataram maior frequência de terem comportamento de risco muito alto para a infecção pelo HIV quando comparados com os demais HSH. Esses resultados provavelmente indicam um elevado número de parcerias sexuais podendo ser fixas, casuais e comerciais e, uso inconsistente de preservativos nas relações sexuais anais com esses parceiros nos 12 meses anteriores $^{29}$. É descrito na literatura ${ }^{12,13,43}$ que o comportamento de risco alto demonstrou estar relacionado à infecção pelo HIV e sífilis, isoladas ou em coinfecção. Diferentemente do encontrado na literatura ${ }^{16}$, nos resultados deste estudo não foi demonstrado associação entre positividade sorológica para o HIV e sífilis à troca de sexo por dinheiro. Ou seja, apesar de estarem mais expostos a diversos fatores de vulnerabilidades ao HIV e à sífilis que os demais, não existe diferença estatística para infecção pelo HIV e sífilis entre os HSH que receberam dinheiro em troca de sexo e os demais HSH da amostra. Sublinha-se que, neste estudo, houve importante número de participantes que não realizou o teste sorológico para o HIV no momento da entrevista, podendo ter gerado um viés na mensuração desta variável.

Sabe-se que, como consequência de fatores socioculturais, estigma, discriminação e violên- 
cia, a população de HSH pode apresentar maior risco de desenvolver transtornos mentais como ansiedade e depressão ${ }^{44}$. Ademais se aponta, na literatura internacional, que alguns tipos de transtornos mentais estão associados ao maior comportamento sexual de risco em $\mathrm{HSH}^{45,46}$. E que, entre os HSH que receberam dinheiro em troca de sexo, altas taxas de depressão e outros transtornos mentais são relatadas em diferentes países ${ }^{16,32,47}$. A depressão e a desesperança estão associadas com a ideação suicida que é considerada um fator de risco para o suicídio efetivo ${ }^{48}$. Este presente estudo, em consonância com a literatura, encontrou associação de forma independente entre ideação suicida com o recebimento de dinheiro em troca de sexo, o que pode expressar maior vulnerabilidade dessa população às condutas autodestrutivas.

Desta forma, destaca-se a importância de programas de prevenção que visem prestar aos $\mathrm{HSH}$, em especial àqueles que receberam dinheiro em troca de sexo, uma assistência humanizada, um acolhimento, uma escuta atenta e aconselhamento psicológico e terapêutico por meio dos serviços de saúde no sentido de evitar que desenvolvam comportamentos e ideações suicidas ou adotem condutas autodestrutivas.

A associação positiva entre a autoidentificação como heterossexual ou bissexual com a troca de sexo por dinheiro entre os HSH brasileiros também é relatada na literatura nacional e internacional ${ }^{13,49}$. A identificação como heterossexual ou bissexual entre os HSH que receberam dinheiro em troca de sexo pode estar associada com menos práticas sexuais anais receptivas do que seus pares homossexuais, sendo portanto, importante fator a se considerar sobre a transmissão do HIV ${ }^{50,51}$. Por outro lado, acredita-se que a associação entre o recebimento de dinheiro em troca de sexo e transtornos mentais possa ser mediada pela identidade sexual, devido ao conflito identitário daqueles que se identificam como heterossexual ou bissexual e o estigma do trabalho sexual entre homens estar relacionada à homossexualidade ${ }^{52}$.

O uso de drogas ilícitas nos seis meses anteriores à entrevista foi associado positivamente à troca de sexo por dinheiro pelos HSH. Este resultado é consistente com a literatura nacional e internacional ${ }^{13,32,49}$. O uso de drogas ilícitas pelos HSH, de um modo geral, é maior que o estimado para a população geral brasileira e esta diferença é mais acentuada entre os $\mathrm{HSH}$ que receberam dinheiro em troca de $\operatorname{sexo}^{13}$. O maior envolvimento com o uso de drogas é elevado entre os HSH que receberam dinheiro em troca de sexo e pode estar relacionado ao estigma, descriminação e violência que estas pessoas são condicionadas ${ }^{49}$. Além disso, os ambientes em que os HSH buscam parceiros sexuais comerciais, devido à marginalização, são frequentemente favoráveis ao consumo de álcool e drogas ilícitas ${ }^{12}$. Outro fator importante é que o recebimento de dinheiro em troca de sexo pode ser resultado de uma necessidade econômica de muitos usuários de drogas ${ }^{53}$.

Neste estudo, ter sofrido violência física devido à orientação sexual esteve associado positivamente com o recebimento de dinheiro em troca de sexo por HSH. As violências devido à orientação sexual são importantes indicadores de vulnerabilidade, pois intimidam, humilham e acarretam em isolamento social, restringindo os locais e horários de circulação no espaço público, assim como o acesso a serviços de saúde, como forma de se preservarem de situações de agressões e/ou de assédio moral.

Este estudo apresenta algumas limitações. Trata-se de um estudo transversal com uma amostra única de HSH brasileiros obtidos de dez cidades de diferentes estados, com diferentes contextos socioculturais, geográficos e epidemiológicos que podem não ser necessariamente representativa de toda a população brasileira de HSH e a técnica de amostragem RDS está potencialmente sujeita a viés de seleção. Desta forma, pode haver heterogeneidade nas características entre os recrutados das amostras de cada cidade. No entanto, teoricamente, quando a amostra atinge o estado de equilíbrio após sucessivas ondas de recrutamento as estimativas obtidas por meio do RDS são robustas e tendem a minimizar este viés ${ }^{27}$. As características da amostra também podem ser influenciadas pela homofilia, ou seja, indivíduos com certas características podem tender a recrutar pares com características semelhantes ${ }^{54}$. No entanto, esse método destacase por atingir as populações de difícil acesso, evitando resultados baseados apenas em amostras de conveniência. Finalmente, apesar da análise combinada das dez amostras independentes não certitificar que os resultados encontrados são representativos da população brasileira de $\mathrm{HSH}$, os dados agrupados originam uma amostra mais robusta, com maior poder estatístico e são mais adequados para o propósito geral de monitoramento de vigilância no âmbito nacional, conforme proposto originalmente. Apesar de tais limitações, a elevada proporção de HSH que trocaram sexo por dinheiro e seus fatores associados apresentados nesse estudo são de grande importância no contexto da saúde pública, no que tan- 
ge à prevenção da infecção pelo HIV e promoção de saúde nesta população.

Conforme demonstrado neste estudo, um elevado número de $\mathrm{HSH}$ relatou ter trocado sexo por dinheiro no ano anterior à entrevista. Os resultados estão em confluência com os achados na literatura nacional e internacional, confirmando a hipótese de que os HSH que receberam dinheiro em troca de sexo apresentam maior vulnerabilidade socioeconômica, programática e comportamental potencialmente aumentando o risco de infecção pelo HIV que os demais $\mathrm{HSH}$ da amostra. As características sociodemográficas e comportamentais de risco, incluindo o uso de drogas ilícitas por esta população demanda o desenvolvimento de estratégias de intervenções específicas que levem em consideração toda a dinâmica social desse grupo, focando na perspectiva dos direitos humanos e no combate ao preconceito, estigma e violência homofóbica.
Diante disso, é necessário que sejam levados em consideração os fatores de vulnerabilidade na construção das políticas de prevenção à infecção pelo HIV e promoção de saúde para os HSH que receberam dinheiro em troca de sexo, também no que diz respeito ao levantamento de informações de maior qualidade sobre essa população. Além das novas abordagens, como a ampliação da testagem para o HIV, o uso da profilaxia pré-exposição (PrEP) ao HIV, a profilaxia após a exposição (PEP), o uso de microbicidas retais, ações de enfrentamento ao estigma e à discriminação destacam-se como importantes instrumentos na redução da incidência de infecções pelo HIV e, consequentemente, sobre o curso da epidemia. Dentre estas, o debate sobre a regulamentação do trabalho sexual, ações publicitárias direcionadas a esse segmento e a promoção da inclusão social desta população ganham destaque no enfrentamento à expansão da epidemia do HIV.

\section{Colaboradores}

DJD Alecrim e MGB Ceccato contribuíram com a concepção do projeto, interpretação dos dados, redação e revisão crítica relevante do conteúdo do artigo, além de acompanhar todas as etapas do trabalho na garantia da exatidão e integridade de qualquer parte da obra. I Dourado, L Kerr e AM Brito contribuíram com a concepção do projeto, redação e revisão crítica relevante do conteúdo do artigo, além de acompanhar todas as etapas do trabalho na garantia da exatidão e integridade de qualquer parte da obra. MDC Guimarães contribuiu com a concepção do projeto, análise e interpretação dos dados, redação e revisão crítica relevante do conteúdo do artigo, além de acompanhar todas as etapas do trabalho na garantia da exatidão e integridade de qualquer parte da obra. 


\section{Referências}

1. Kerr LS, Mota RS, Kendall C. Pinho AA, Mello MB, Guimarães MDC, Dourado I, Brito AM, Benzaken A, McFarland W, Rutherford G. HIV among MSM in a large middle-income country. AIDS 2013; 27(3):427435.

2. Brasil. Ministério da Saúde (MS). Boletim Epidemiológico AIDS e DST. Brasília: MS; 2015.

3. Brasil. Ministério da Saúde (MS). Pesquisa de conhecimento, atitudes e práticas na população brasileira. Brasília: MS; 2011.

4. Barbosa Junior A, Szwarcwald CL, Pascom AR, Souza Júnior PB. Tendências da epidemia de AIDS entre subgrupos sob maior risco no Brasil, 1980-2004. Cad Saude Publica 2009; 24(4):727-737.

5. Reisner SL, Mimiaga MJ, Mayer KH, Tinsley JP, Safren SA. Tricks of the trade: sexual health behaviors, the context of HIV risk, and potential prevention intervention strategies for male sex workers. J LGBT Health Res 2008; 4(4):195-209.

6. Bacon O, Lum P, Hahn J, Evans J, Davidson P, Moss A, Page-Shafer K. Commercial sex work and risk of HIV infection among young drug-injecting men who have sex with men in San Francisco. Sex Transm Dis 2006; 33(4):228-234.

7. Montano SM, Sanchez JL, Laguna-Torres A, Cuchi P, Avila MM, Weissenbacher M, Serra M, Viñoles J, Russi JC, Aguayo N, Galeano AH, Gianella A, Andrade R, Arredondo A, Ramirez E, Acosta ME, Alava A, Montoya O, Guevara A, Manrique H, Sanchez JL, Lama JR, de la Hoz F, Sanchez GI, Ayala C, Pacheco ME, Carrion G, Chauca G, Perez JJ, Negrete M, Russell KL, Bautista CT, Olson JG, Watts DM, Birx DL, Carr JK; South American HIV Molecular Surveillance Working Group. Prevalences, genotypes, and risk factors for HIV transmission in South America. J Acquir Immune Defic Syndr 2005; 40(1):57-64.

8. Lama JR, Lucchetti A, Suarez L, Laguna-Torres VA, Guanira JV, PunM, Montano SM, Celum CL, Carr JK, Sanchez J, Bautista CT, Sanchez JL. Association of herpes simplex virus type 2 infection and syphilis with human immunodeficiency virus infection among men who have sex with men in Peru. J Infect Dis 2006; 194(10):1459-1466.

9. Valderrama MBM, Carcamo C, Garcia P, Bernabe A, Cotrina A, Chiappe M, Guerra C, Gonzales M, Garnett G, Espinosa B, Gadea N, Montano S, Nieto M, Holmes K. High HIV and syphilis prevalence among male commercial sex workers from the Peruvian Amazon. International AIDS Conference; 2008; Mexico City, Mexico.

10. Bayer AM, Garvich M, Diaz DA, Sanchez H, Garcia PJ, Coates TJ. When Sex Work Becomes Your Everything: The Complex Linkages Between Economy and Affection Among Male Sex Workers in Peru. Am J Mens Health 2014; 8(5):373-386.

11. Ramos Farias MS, Garcia MN, Reynaga E, Romero M, Vaulet ML, Fermepin MR, Toscano MF, Rey J, Marone R, Squiquera L, González JV, Basiletti J, Picconi MA, Pando MA, Avila MM. First report on sexually transmitted infections among trans (male to female transvestites, transsexuals, or transgender) and male sex workers in Argentina: high HIV, HPV, HBV, and syphilis prevalence. Int J Infect Dis 2011; 15(9):e635640.
12. Chao GF. Prostituição masculina, HIV/AIDS: estudo epidemiológico em municípios do Ceará [dissertação]. Fortaleza: Universidade Federal do Ceará; 2008.

13. Tun W, Mello M, Pinho A, Chinaglia M, Diaz J. Sexual risk behaviours and HIV seroprevalence among male sex workers who have sex with men and non-sex workers in Campinas, Brazil. Sex Transm Infect 2008; 84(6):455-457.

14. Joint United Nations Programme on HIV/AIDS (UNAIDS). Technical guidance for Global Fund HIV proposals Round 11. New York: UNAIDS; 2011.

15. Joint United Nations Programme on HIV/AIDS (UNAIDS). Sex work and HIV/AIDS. Technical Update. Geneva: UNAIDS. 2002.

16. Baral SD, Friedman MR, Geibel S. Male sex workers: practices, contexts, and vulnerabilities for HIV acquisition and transmission. Lancet 2015; 385(9964):260273.

17. Caceres CF, Bayer AM, Gomero A, Grenfell P, Salazar X. Men who sell sex in Peru: evolving technological and sexual cultures. Aggleton P, Parker R, editors. Men Who Sell Sex: Global Perspectives. London: Taylor and Francis; 2015.

18. Mimiaga MJ, Reisner SL, Tinsley JP, Mayer KH, Safren SA. Street workers and internet escorts: contextual and psychosocial factors surrounding HIV risk behavior among men who engage in sex work with other men. J Urban Health 2009; 86(1):54-66.

19. Rigoletto RN. Um olhar sobre a prostituição masculina. In: XII CLASES; 2004; Santiago.

20. Santos MA. Prostituição Masculina e Vulnerabilidade às DSTS/AIDS. Texto Contexto Enferm 2011; 20(1):7684 .

21. Abreu W. O submundo da prostituição, vadiagem e jogo do bicho. $16^{\mathrm{a}}$ ed. Rio de Janeiro: Record; 1998.

22. Passos AD, Figueiredo JF. Risk factors for sexually transmited diseases in prostitutes and transvestites in Ribeirão Preto (SP), Brazil. Rev Panam Salud Publica 2004; 16(2):95-101.

23. Brasil. Ministério da Saúde (MS). Plano nacional de enfrentamento da epidemia de AIDS e das DST entre gays, HSH e travestis. Brasília: MS; 2007.

24. Padilla MB, Guilamo-Ramos V, Bouris A, Reyes AM. HIV/AIDS and tourism in the Caribbean: an ecological systems perspective. Am J Public Health 2010; 100(1):70-77.

25. Liu H, Liu H, Cai Y, Rhodes AG, Hong F. Money boys, HIV risks, and the associations between norms and safer sex: a respondent-driven sampling study in Shenzhen, China. AIDS Behav 2009; 13(4):652-662.

26. Brasil. Ministério da Saúde (MS). 'UNGASS - HIV/ Aids, Resposta Brasileira 2008-2009. Relatório de Progresso do País. Brasilia: MS; 2010.

27. Heckathorn DD. Respondent-driven sampling: a new approach to the study of hidden populations. Soc Probl 1997; 44(2):174-199.

28. Kerr LRFS. Comportamento, atitudes, práticas e prevalência de HIV e sifilis entre homens que fazem sexo com homens (HSH) em 10 cidades brasileiras [relatório]. Brasília: Ministério da Saúde (MS); 2009.

29. Rocha GM. Comportamento sexual de risco entre homens que fazem sexo com outros homens no Brasil [tese]. Belo Horizonte: Universidade Federal de Minas Gerais; 2014. 
30. Szwarcwald CL, Souza JRPRB, Damacena GN, Barbosa Júnior A, Kendall C. Analysis of Data Collected by RDS Among Sex Workers in 10 Brazilian Cities, 2009: Estimation of the Prevalence of HIV, Variance and Design Effect. J Acquir Imune Defic Syndr 2011; 57(Supl. 3):S129-S135.

31. Myers T, Allman D, Xu K, Remis RS, Aguinaldo J, Burchell A, Calzavara L, Swantee C. The prevalence and correlates of hepatitis $\mathrm{C}$ virus (HCV) infection and HCV-HIV co-infection in a community sample of gay and bisexual men. Int J Infect Dis 2009; 13(6):730-739.

32. Weber AE, Craib KJ, Chan K, Martindale S, Miller ML, Schechter MT, Hogg RS. Sex trade involvement and rates of human immunodeficiency virus positivity among young gay and bisexual men. Int J Epidemiol 2001; 30(6):1449-1454.

33. Gorbach PM, Murphy R, Weiss RE, Hucks-Ortiz C, Shoptaw S. Bridging sexual boundaries: men who have sex with men and women in a street-based sample in Los Angeles. J Urban Health 2009; 86(Supl. 1):63-76.

34. Newman PA, Rhodes F, Weiss RE. Correlates of sex trading among drug-using men who have sex whit men. Am J Public Health 2004; 94(11):1998-2003.

35. Nerlander LM, Hess KL, Sionean C, Rose CE, Thorson A, Broz D, Paz-Bailey G. Exchange Sex and HIV Infection Among Men Who Have Sex with Men: 20 US Cities, 2011. AIDS Behav 2016; 21(8):2283-2294.

36. Beyrer C, Crago AL, Bekker LG, Butler J, Shannon K, Kerrigan D, Strathdee SA. An action agenda for HIV and sex workers. Lancet 2014; 6736(15):1-14.

37. Kelly JA, Amirkhanian YA, Mcauliffe TL, Dyatlov RV, Granskaya J, Borodkina OI. HIV risk behavior and risk-related characteristics of young Russian men who exchange sex for money or valuables from other men. AIDS Education and Prevention 2001; 13(2):175-88.

38. Ballester-Arnal R, Gil-Llario MD, Salmeron-Sanchez P, Gimenez-Garcia C. HIV prevention interventions for young male commercial sex workers. Current HIV/AIDS reports 2014; 11(1):72-80.

39. Paiva V, Calazans G, Venturi G, Dias R. Idade e uso de preservativos na iniciação sexual de adolescentes brasileiros. Rev Saude Publica 2008; 42(Supl 1):45-53.

40. Teixeira AMFB, Knauth DR, Fachel JMG, Leal AF. Adolescentes e uso de preservativos: as escolhas de jovens de três capitais brasileiras na iniciação e na última relação sexual. Cad Saude Publica 2006; 22(7):1385-1396.

41. Beymer MR, Weiss RE, Bolan RK, Rudy ET, Bourque LB, Rodriguez JP. Sex on demand: geosocial networking phone apps and risk of sexually transmitted infections among a cross-sectional sample of men who have sex with men in Los Angeles County. Sex Transm Infect 2014; 90(7):567-572.

42. Grosskopf NA, Levasseur MT, Glaser DB. Use of the internet and mobile-based "apps" for sex-seeking among men who have sex with men in New York City. Am J Mens Health 2014; 8(6):510-520.

43. Mattson CL, Campbell D, Karabatsos G, et al. Scaling sexual behavior or 'sexual risk propensity' among men at risk for HIV in Kisumu, Kenia. AIDS Behav 2010; 14(1):162-172.

44. Nguyen, HM. Understanding Male Sex Work: A Literature Review. J Subst Abuse Alcohol 2017; 5(1):1054.
45. Safren SA, Thomas BE, Mimiaga MJ, Chandrasekaran V, Menon S, Swaminathan S, Mayer KH. Depressive symptoms and human immunodeficiency virus risk behavior among men who have sex with men in Chennai, India. Psychology, Health and Medicine 2009; 14(6):705-715

46. Safren SA, Reisner SL, Herrick A, Mimiaga MJ, Stall RD. Mental health and HIV risk in men who have sex with men. J Acquir Immune Defic Syndr 2010; 55(Supl.):S74-S77.

47. Goldsamt LA, Clatts MC, Giang LM, Yu G. Prevalence and behavioral correlates of depression and anxiety among male sex workers in Vietnam. Intern J Sex Health 2014; 27(2):145-155.

48. Borges VR, Werlang BSG. Estudo de ideação suicida em adolescentes de 15 a 19 anos. Estudos de Psicologia (Natal) 2006; 11(3):345-351.

49. Ross MW, Timpson SC, Williams ML, Amos C, Bowen A. Stigma consciousness concerns related to drug use and sexuality in a sample of street-based male sex workers. Int J Sex Health 2007; 19(2):57-65.

50. Elifson KW, Boles J, Sweat M. Risk factors associated with HIV infection among male prostitutes. $\mathrm{Am} \mathrm{J} \mathrm{Pu-}$ blic Health 1993; 83(1):79-83.

51. Boles J, Elifson KW. Sexual identity and HIV: The male prostitute. The Journal of Sex Research 1994; 31:39-46.

52. Bar-Johnson M, Weiss P. Mental health and sexual identity in a sample of male sex workers in the Czech Republic. Med Sci Monit 2014; 20:1682-1686.

53. Van Den Hoek JAR, Coutinho RA, Van Haastrecht HJA, Van Zadelhoff AW, Goudsmit J. Prevalence and risk factors of HIV infections among drug users and drug-using prostitutes in Amsterdam. AIDS 1988; 2(1):55-60.

54. Gile KJ, Handcock MS. Respondent-driven sampling: as assessment of current methodology. Sociol Methodol 2010; 40(1):285-327.

Artigo apresentado em 21/03/2018

Aprovado em 14/07/2018

Versão final apresentada em 16/07/2018 
